\title{
Radiation Exposure to the Physician in Interventional Pain Management
}

\author{
Laxmaiah Manchikanti, MD*, Kim A. Cash, RT", Tammy L. Moss, RT" and Vidyasagar Pampati, MSc**
}

Fluoroscopy is an integral part of the practice of interventional pain management in present day modern medical practices. The major purpose of fluoroscopy in interventional pain management is correct needle placement to ensure target specificity and accurate delivery of the injectate. Fluoroscopy has become mandatory for multiple procedures based either on the definition of the procedure or the requirement of third parties. The most commonly used fluoroscopy in interventional pain management is with $\mathrm{C}$-arm fluoroscopes with image intensification. Fluoroscopy is associated with risk for patients, clinicians, and the personnel in the operating room unless it is managed with appropriate understanding, skill, and vigilance.

A total of 1,000 consecutive patients undergoing interventional procedures with chronic pain performed by one physician were studied. Two fluoroscopy units were utilized and operated by two certified radiological technologists. The procedures performed included caudal and interlaminar epidural injections, facet joint nerve blocks, percutaneous adhesiolysis, intercostal nerve blocks, sympathetic blocks, transforaminal epidural injections, along with other procedures. Results showed that a total of 1,000 patients underwent 1,729 procedures with average radiation exposure of $13.2 \pm 0.33$ seconds per patient and $7.7 \pm 0.21$ seconds per procedure. Dosimetry measurements showed total exposure of 1,345 mREM outside the apron and 0 mREM inside the apron during this period. The average exposure outside the apron was 1.345 mREM per patient and $0.748 \mathrm{mREM}$ per procedure outside the apron and 0 mREM inside the apron. The levels of exposure are significantly below the annual limits recommended. It is concluded that it is feasible to perform all procedures under fluoroscopy in the described setting safely and effectively in interventional pain management.

Keywords: Interventional pain management, fluoroscopy, $\mathrm{C}$-arm, computed tomographic fluoroscopy, radiation exposure, radiation toxicity, scatter, maximum permissible dose, as low as reasonably achievable (ALARA)
Fluoroscopy has been an integral part of the practice of diagnostic radiology since the early twentieth century. There has been an explosion in the use of fluoroscopic modalities across all disciplines of medicine in recent years. The discipline of interventional pain management is no exception to this trend. While the previous experience of fluoroscopic procedures was limited to diagnosis with relatively small risk to the physician, patient and other personnel, recent explosion of utilization of fluoroscopy

From Pain Management Center of Paducah, Paducah, Kentucky. *Dr. Manchikanti is the medical director, \#Ms. Cash and Ms. Moss are radiological technologists, and **Mr. Pampati is the statistician at Pain Management Center of Paducah. Address correspondence: Laxmaiah Manchikanti, MD, 2831 Lone Oak Road, Paducah, KY 42003. E-mail: drm@apex.net

Funding: No external financial support was provided. in almost all interventional procedures, has extended to therapeutic modalities, with increased risk. It has been estimated that over 1,000,000 interventional procedures are performed annually in the United States to manage chronic pain, with at least $50 \%$ of them being performed under fluoroscopy. The dramatic increase in the use of imaging modalities and their promise in improving diagnosis and treatment in chronic pain has been recognized (1-16). This has resulted in increased number of publications highlighting the usefulness of imaging techniques in pain management. Fluoroscopy offers many advantages for guiding interventional procedures. Most interventional procedures in the management of chronic pain require fluoroscopic exposure only for short periods of time. The modern developments with complex procedures being performed percutaneously have resulted in the number of prolonged fluoroscopic procedures. The major purpose of fluoroscopy in interventional pain management is correct needle placement to ensure target specificity and accurate delivery of the injectate (3-25). 
Incorrect needle placement has been described for multiple procedures without fluoroscopy (17-25). In fact, fluoroscopy has become mandatory for multiple procedures based on the definition of the procedure or requirement of third parties (10-14). The most commonly used fluoroscopy in interventional pain management is with $\mathrm{C}$-arm fluoroscopes with image intensification. However, computed tomographic (CT) fluoroscopy is also being increasingly used to perform these procedures.

Fluoroscopy is associated with risk for patients, clinicians, and the personnel in the operating room unless it is managed with appropriate understanding, skill, and vigilance (3). Fishman et al (3) described that risks can be minimized with a healthy respect for electromagnetic radiation, continued radiation safety education, radiation monitoring, and safe "common sense" practices, along with keeping in mind basic principles of ALARA (as low as reasonably achievable) time, distance, and shielding. The primary health risks to physician, patient, and staff in interventional pain management setting exposed to radiation include cancer, cataract formation, and fetal deformities among other complications, including radiation burns (26). Most risks in interventional pain management, specifically to the physician and staff originate from the scatter. Scatter is the radiation that bounces off the patient. The US Food and Drug Administration (FDA) has reported 50 cases of radiation injuries to the patients from fluoroscopically guided procedures in 1994 alone (27). It was also shown that physicians who performed radiography and fluoroscopy in the first half of the twentieth century had higher rates of cancer-related deaths than any other group of physicians (28). The FDA in 1994 issued an advisory regarding the need for proper training for all personnel involved with fluoroscopy (29). Thus, minimization of exposure is crucial in interventional pain management similar to other settings. The radiation dose depends on the type of the procedure, the patient's size, the equipment, the technique, and many other factors. One of the major concerns with use of CT fluoroscopy is its high radiation exposure $(26,30)$. However, radiation exposure may be reduced by using intermittent exposures, grid removal, last image hold, dose spreading, beam filtration, pulsed fluoroscopy, and other dose reduction techniques (26).

Multiple studies have been performed to evaluate radiation exposure to orthopedic surgeons, cardiologists, urologists, orthopedic surgeons and interventional radiologists performing a variety of visceral and peripheral angiographic procedures (26). However, there have been only a few reports published evaluating the exposure in interventional pain management $(15,16,30)$. These studies evaluated a small number of procedures and were highly variable. Botwin et al $(15,16)$ evaluated the radiation exposure of a spinal interventionalist performing either fluoroscopically guided lumbar transforaminal epidural steroid injections or fluoroscopically guided caudal epidural steroid injections in which radiation exposure ranged from 2 to 38 seconds. Paulson et al (30) in investigating radiation dose to radiologists with $\mathrm{CT}$ fluoroscopy-guided interventional procedures, showed that fluoroscopic time varied from 11 seconds (6.2 to 19.2) for sacroiliac joint injection, 18.4 seconds (12.0 to 27.6) for cervical injections, and 17.6 seconds (3.6 to 65.0) for lumbar injections.

It appears that fluoroscopic times for interventional procedures are much less than a multitude of procedures typically involving extended fluoroscopic exposure time including percutaneous transluminal angioplasty, vascular embolization, percutaneous nephrostomy, etc., which ranged from 7 minutes for nephrostomy to 14.6 minutes for percutaneous transhepatic cholangiography and as high as 34.1 minutes for cerebral embolization (26). In spite of the safety described due to short exposure time in interventional pain management, risks could be increased proportionately based on using continuous fluoroscopy, not following appropriate precautions, poor training, and overenthusiasm. This prospective evaluation was undertaken to monitor multiple procedures performed on consecutive patients over a period of 4 months in an ambulatory surgery center setting by a single physician.

\section{METHODS}

A total of 1,000 consecutive patients undergoing interventional procedures with chronic pain by a single physician were included. The study was performed at a non-university interventional pain management practice, procedures being performed in a sterile environment in an ambulatory surgery center. Two fluoroscopy units were utilized and operated by two certified radiological technologists. Inclusion criteria consisted of consecutive patients presenting for either diagnostic or therapeutic fluoroscopically guided interventional procedures. Exclusion criteria included allergy to iodine or any component of the injection. Multiple procedures or previous surgery were not considered as exclusion.

Most procedures were performed in the prone position except for cervical sympathetic blocks and intraarticular injections of acromioclavicular joint. The procedures were performed in one of the two operating rooms with OEC 
fluoroscopic units, either Compact 7600 or Compact 7800 OEC, Salt Lake City, Utah. The procedures varied from facet joint nerve blocks to spinal endoscopic adhesiolysis. Procedures were performed in a Posterior-Anterior view and a lateral fluoroscopic view was utilized to confirm the needle position when it was deemed necessary. The mode of fluoroscopy utilized ranged from pulsed-imaging for medial branch blocks to rare continuous fluoroscopic imaging to rule out intravascular injections, as well as with discography and transforaminal epidural injections.

Data were collected on patient demographics, types of the procedures and number of regions treated in each patient, and variable fluoroscopic time based on each procedure and total exposure time for each patient.

The SPSS version 9.0 statistical package was used to generate frequency tables. Results were considered statistically significant if the $p$ value was less than 0.05 .

\section{RESULTS}

Table 1 illustrates demographic features with gender, age, height and weight, body mass index, number of regions treated and number of procedures performed.
Table 1. Demographic features

\begin{tabular}{lc}
\hline & Total / Average \\
\hline Total number of patients & 1000 \\
Male & 378 \\
Female & 622 \\
Age (yrs) (mean \pm SEM) & $49.5 \pm 0.45$ \\
Weight (lbs) (mean \pm SEM) & $179 \pm 1.46$ \\
Height (inches) (mean \pm SEM) & $66.6 \pm 0.12$ \\
Body mass index (mean \pm SEM) & $28.5 \pm 0.22$ \\
\hline
\end{tabular}

Table 2 illustrates radiation exposure in all patients showing per patient exposure, per procedure exposure, along with exposure for each type of procedures individually, along with total exposure times.

\section{DISCUSSION}

A total of 1,000 patients were treated with 1,729 interventional pain management procedures by a single physician in an ambulatory surgery center. Our results showed that total exposure for 1,729 procedures in 1,000

Table 2. Illustration of radiation exposure in 1000 patients

\begin{tabular}{lccc}
\hline & Number & $\begin{array}{c}\text { Radiation Expos ure in } \\
\text { Seconds }\end{array}$ & Range \\
\hline Per Procedure & - & $7.7 \pm 0.21$ & $1-69$ \\
Per Patient & - & $13.2 \pm 0.33$ & $1-97$ \\
Cervical Facet Joint Nerve Blocks & 547 & $5.9 \pm 0.07$ & $1-19$ \\
Cauda/Interlaminal Epidurals & 353 & $3.75 \pm 0.13$ & $1-27$ \\
Lumbar Facet Joint Nerve Blocks & 352 & $5.7 \pm 0.09$ & $1-14$ \\
Intercostal Nerve Blocks & 96 & $4.0 \pm 0.18$ & $1-8$ \\
Percutaneous Adhesiolysis & 85 & $18.9 \pm 1.32$ & $4-45$ \\
Lumbar Transforaminal Epidurals & 72 & $10.9 \pm 0.72$ & $3-32$ \\
Thoracic Facet Joint Nerve Blocks & 68 & $5.1 \pm 0.33$ & $1-13$ \\
Joint Injections & 67 & $7.5 \pm 1.28$ & $1-52$ \\
Cervical Transforaminal Epidurals & 28 & $8.8 \pm 0.78$ & $3-17$ \\
Stellate Ganglion Block(s). & 20 & $6.5 \pm 0.68$ & $2-11$ \\
Lumbar Sympathetic Block(s) & 17 & $12.0 \pm 1.49$ & $6-27$ \\
Medial Branch Neurotomy & 16 & $12.7 \pm 1.49$ & $6-23$ \\
Spinal Endoscopy & 8 & $39.6 \pm 2.31$ & $25-46$ \\
\hline
\end{tabular}


patients was 13,200 seconds. The average per patient was $13.2 \pm 0.33$ seconds of radiation with a range of 1 to 97 seconds. However, some patients received multiple procedures with an average of 2 procedures per patient. Per procedure radiation exposure in seconds was $7.7 \pm$ 0.21 . Table 2 illustrates radiation exposure in seconds for multiple procedures ranging from 25 to 46 seconds for spinal endoscopy to 1 to 13 seconds for thoracic facet joint nerve blocks. The radiation exposure to the physician at the outside apron over the upper part of the chest was $1,345 \mathrm{mREM}$ for the entire period with an average of 1.345 mREM per patient (deep-dose equivalent) or $0.748 \mathrm{mREM}$ per procedure (deep-dose equivalent). However, the inside apron over the thyroid was $0 \mathrm{mREM}$ deep dose equivalent. This evaluation illustrates the importance of using protective measures and keeping in mind the basic principles of ALARA (as low as reasonably achievable), time, distance, and shielding. This may reduce or eliminate primary health risks to physician, patient, and staff in interventional pain management setting exposed to radiation. Our results are similar to Botwin et al $(15,16)$ showing the radiation exposure of a spinal interventionalist performing either fluoroscopically guided lumbar transforaminal epidural steroid injections or fluoroscopically guided caudal epidural steroid injections in which radiation exposure ranged from 2 to 38 seconds. Botwin et al $(15,16)$ showed that radiation exposure of the spinal interventionalist performing lumbar transforaminal epidural steroid injections ranged from 5 to 38 seconds with an average of 15.6 seconds. They (16) also showed that while performing caudal epidural steroid injections, the range was 2 to 33 seconds with mean fluoroscopy time of 12.55 seconds. Paulson et al (30) showed that mean fluoroscopic time ranged from 11 seconds for sacroiliac joint injection, 18.4 seconds for cervical injections and 17.6 seconds for lumbar injections. They also showed that no radiation was detectable at the inside apron or at the outside room control badge. Thus, our findings correlate with the findings of Botwin et al $(15,16)$, as well as Paulson et al (30). This study also includes a large number of patients undergoing multiple procedures.

This evaluation may be criticized for not using multiple badges at various levels as others have performed in the past on the physician, as well as the patient. However, no matter how many monitors are utilized over a physician or the patient, the exposure apparently remains to be an important factor which needs to be minimized. As this study shows, the radiation exposure can be minimized to a significant degree utilizing appropriate precautions. Our study also showed patients with higher body mass index
(BMI) required larger radiation doses. As shown in Fig. 1, non-obese showed mean radiation of 12.2 seconds per patient and 7.4 seconds per procedure in contrast to obese patients with a requirement of radiation exposure of 14.9 seconds per patient or 8.9 seconds per procedure with significant differences $(p=0.007)$ between obese and nonobese patients. This study also judges fluoroscopic timings based on total patient exposure rather than per procedure. This is important as many patients in interventional pain management setting present with involvement of multiple regions requiring treatment of multiple regions at the same time (40). Thus, this study again demonstrates that if appropriate techniques are used and multiple dose reduction techniques are utilized, procedures can be performed safely under fluoroscopy without risk to the patient, physician, and the personnel. This is shown by the average fluoroscopy time of 13.2 seconds per patient or 7.7 seconds per procedure extrapolated to 2,000 or 3,000 procedures will lead to fluoroscopic exposure of 15,400 seconds or 23,100 seconds. Based on this evaluation with 1.345 mREM outside the apron with 0 mREM inside the apron, in a worse case scenario, a physician will be exposed to 4,035 mREM on outside and 0 mREM inside the apron for 3,000 procedures. Considering the annual limit for whole body exposure of 5 REM per year total effective dose equivalent, if no protection is used, an interventionalist performing 4,000 procedures will still be at less than maximum level. Further, general radiation exposure guides to lens of the eye are 15 REM per year and extremities and skin are 50 REM per year. However, inside the apron was shown to be 0 REM under the circumstances described. Hence,

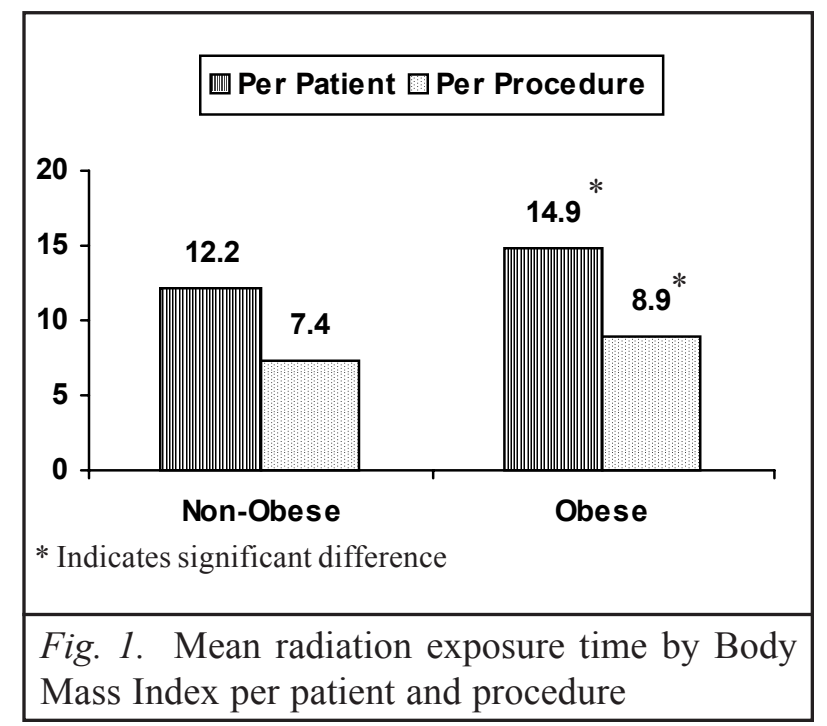


under these circumstances, it can be stated that performing interventional procedures under fluoroscopy is not only feasible but also safe.

Fishman et al (3) described that pain specialists frequently employ fluoroscopy to guide injection procedures. They cautioned that while fluoroscopy offers many advantages for guiding analgesic procedures, it does not come without risk for patients or clinicians if it is managed without understanding and vigilance. Rathmell (1) described that many recent articles have delineated the usefulness of fluoroscopy in performing guided injection techniques. Rathmell (1) also stated that the majority of pain practitioners who perform any sort of injection techniques as part of their practices have quickly learned the advantages of fluoroscopy in guiding precise needle placement. However, it appears that in spite of the widening use of fluoroscopy and other imaging modalities in diagnosis and treatment, the curricula in pain management training programs have lagged behind (1). There is no formal requirement for education of pain physicians about the use of diagnostic imaging and the evaluation and treatment of the pain patient within accredited pain fellowship programs (1). While most training programs in pain management, as well as other disciplines of medicine have increased instruction of their trainees in the use of fluoroscopy, the radiation safety aspect is often omitted entirely (1). Rathmell (1) raised important questions: How does the radiation exposure of the typical pain practitioner, who routinely uses fluoroscopy compare with that of other practitioners using fluoroscopy? What have these other specialists learned about the risks of cumulative radiation exposure using modern guidelines for minimizing exposure? Further, he also questioned pain practitioners, if we should even be concerned about radiation exposure when specialists like invasive cardiology colleagues use magnitudes more radiation in performing invasive cardiology interventions under biplanar cineangiography? In fact, Mahesh (26) published extensively on this issue. He examined various radiation exposures during the fluoroscopic procedures and various dose reduction techniques emphasizing the importance of training for operators of fluoroscopic systems. The FDA Public Health Advisory (29) in 1994 identified multiple procedures typically involving extended fluoroscopic exposure time. None of these included interventional pain management procedures. However, in 1994, fluoroscopy for interventional pain management was not in such widespread use. Shope (27) described that an estimated 300,000 coronary angioplasty procedures were performed in the United States in 1994. Conservative estimations project this in 2001, 2.5 to 3 times the 1994 estimation (26). Along with pain management, a wide range of other fluoroscopically guided procedures, such as neuroembolization, placement of transjugular intrahepatic portosystemic shunts, have also shown considerable growth. Thus, considering the nature of these prolonged fluoroscopic procedures, and increasing reports of high skin doses causing significant tissue injury has emerged (26). Since 1994, the FDA has documented many cases of radiation-induced burns (31), along with a number of case histories of injuries to both patients and physicians appearing in the literature (32-37). It also has been described that some of the radiation-induced wounds have required skin grafts, resulting in permanent disfigurement, along with numerous other side effects including malignancy. However, the actual extent of the problem continues to be unknown. This is partly fueled by lack of requirements for reporting and lack of a central repository for this information.

Biologic effects of radiation can be broadly grouped as stochastic or non-stochastic effects. A stochastic effect is one in which the probability of the effect, rather than its severity, increases with dose (26). Thus, among the multiple effects of radiation, cancer and genetic effects are considered as stochastic. Stochastic effect is exemplified by the fact that the probability of radiationinduced leukemia is substantially greater after exposure to $100 \mathrm{rad}$ than after exposure to $1 \mathrm{rad}$. However, if leukemia does occur, there is no difference in the severity of the disease. It is believed that stochastic effects lack a threshold dose because injury to a few cells or even a single cell could theoretically result in production of the effect (26). In contrast, for non-stochastic or deterministic effects, the probability of causing certain types of harm will be zero at small radiation doses. For effects such as cataracts, erythema, epilation, and even death which are considered as non-stochastic or deterministic effects that can result from high radiation exposures, damage will become apparent, only above the threshold levels and severity also increases as dose rises above the threshold. Table 3 illustrates general radiation exposure limits. For interventional pain management procedures, at diagnostic x-ray energies (i.e., those for fluoroscopy and computed tomography) doses are higher at the beam entrance point, the most commonly observed harm has been skin tissue damage and hair loss $(31,37)$. Fortunately, modern fluoroscopy systems based on image intensifiers are extremely flexible devices and permit operation in a wide range of modes for dynamic and static imaging (26). Yet, accompanying this flexibility is the fact that different 
Table 3. Annual maximum target area / organ permissible radiation doses

\begin{tabular}{ll}
\hline Area/Organ & $\begin{array}{l}\text { Annual Maximum } \\
\text { Permissible Dose }\end{array}$ \\
\hline Thyroid & $50 \mathrm{rem}(500 \mathrm{mSv})$ \\
Extremities & $50 \mathrm{rem}(500 \mathrm{mSv})$ \\
Lens of the eye & $15 \mathrm{rem}(150 \mathrm{mSv})$ \\
Gonads & $50 \mathrm{rem}(500 \mathrm{mSv})$ \\
Whole body & $5 \mathrm{rem}(50 \mathrm{mSv})$ \\
Pregnant women & $0.5 \mathrm{rem}$ to fetus $(5 \mathrm{mSv})$ \\
\hline
\end{tabular}

Adapted and modified from NCRP report (38).

imaging modes have different dose characteristics making implementation of safety a difficult task. Fluoroscopes typically have the capability of operation in a number of dynamic imaging modes: normal fluoroscopy, high-dose fluoroscopy, and conventional and digital cinefluoroscopy (26). Thus, physician and the operator should have the knowledge of the relative dose characteristics of the different imaging modes. Even though there are a multitude of factors, the performance of a fluoroscopy system with respect to radiation dose is best characterized by the receptor entrance exposure rates and skin entrance exposure rates (26). Further, exposure rates are dependent on the patient thickness and operational factors.

The likelihood of deterministic radiation skin injury to a patient or physician is predictable from the skin dose. The skin entrance exposure is measured to determine its maximum value, based on regulations by various agencies in fluoroscopic mode (26). The skin entrance exposure limitations set by regulatory bodies are $2.58 \mathrm{mC} / \mathrm{kg}$ per minute $(10 \mathrm{R} / \mathrm{min})$ for normal fluoroscopy and $5.16 \mathrm{mC} / \mathrm{kg}$ per minute $(20 \mathrm{R} / \mathrm{min})$ for high-dose fluoroscopy. However, currently there are no maximum limitations on the fluoroscopic imaging modes, such as cine and digital substraction angiography.

No amount of radiation can be considered safe to the patient, physician, or other personnel. The maximum permissible dose (MPD) is the dose at the upper limit of radiation that one should be "allowed" to receive without substantial risk of clinically significant adversity. Radiation doses below this level probably only carry a remote chance of clinically significant adverse effects $(38,39)$. The annual effective whole body dose limit for physicians is $50 \mathrm{msv}$ or 5REM (38). Fishman et al (3) described that assuming proper technique and equipment and that the clinician is more than one meter away from the patient, the scattered radiation exposure to the practitioner for a lumbar epidural steroid injection performed under fluoroscopy guidance measured was as low as 0.03 mREM. Botwin et al $(15,16)$ extrapolated their data to 1,000 fluoroscopically guided caudal epidural steroid injections with a total radiation exposure varying from $2.44 \mathrm{mREM}$ at the glasses to 4.10 mREM to the finger; and average exposure for transforaminal epidural steroid injection for 100 procedures was $70 \mathrm{mREM}$ at the ring badge, $40 \mathrm{mREM}$ at the glasses badge and $30 \mathrm{mREM}$ at the outside apron badge. They also noted that the radiographic technologist's average exposure during these procedures was below the limit of detectability. However, these exposures described in the literature are relatively small and should not lead the interventional practitioner into a false sense of security. It is also imperative to remember that radiation dose is cumulative. Even though fluoroscopic radiation exposure in interventional pain management is small, with improper use it could be fatal.

To maintain radiation safety, multiple techniques have been described to limit skin doses during interventional procedures. These involve either methodological or technical features present in the equipment. The Nuclear Regulatory Commission (NRC) and most other radiation safety agencies endorse the concept of ALARA (as low as reasonably achievable), which is based on the premise that all exposures that can be prevented should be prevented. Fishman et al (3) described three major steps to reduce clinician exposure to scattered radiation, which included distance to, time of, and protection from radiation. Mahesh (26) described multiple dose reduction techniques which included intermittent fluoroscopy, grid removal, last image hold, dose spreading, beam filtration, pulsed fluoroscopy and other dose reduction techniques, including proper training of fluoroscopy operators.

Maximizing distance from the radiation source is an obvious, but often overlooked means of decreasing the risk. The major source of radiation is the patient who serves as a conduit for scattered radiation (3). Generally, the fluoroscopic scatter exposure level from patients at 1 $\mathrm{m}$ is roughly $0.1 \%$ of the entrance skin exposure (41). The inverse square law describes the significant decrease of exposure with increased distance from the source, thereby doubling the distance quarters the exposure (42). However, 
interventional practitioners should be aware, especially when employing a crosstable lateral view with a C-arm unit, that the amount of scattered radiation may be two to three times higher at the entrance surface of the patient i.e., x-ray tube than at the exit surface of the patient, i.e., image intensifier (43). Thus, as a practical point, standing on the image intensifier side of the patient will offer less exposure (3). Further, maintaining the image intensifier as close to the patient as possible also reduces exposure. Maintaining the x-ray source to tabletop distance at as large a distance as practical reduces the entrance exposure substantially for a given exposure to the image intensifier detector (3).

Intermittent fluoroscopy (i.e., keeping the x-rays on only a few seconds at a time), long enough to view the current position of the needle, can reduce total fluoroscopic times considerably. Mahesh (26) described that this simple technique is particularly effective when combined with last image hold features.

The presence of grids in $\mathrm{x}$-ray systems primarily increases the contrast and hence, the image quality. However, they also increase the dose to the patient and staff by a factor of two or more (26). Studies have shown that, removal of the grid has resulted in dose reduction of up to one third to one half with little or no degradations in contrast and image quality $(44,45)$.

Last image hold and electronic collimation have been described as dose reduction and saving features. Last image hold is a saving feature (46), since it allows physicians to contemplate the last image and plan the next move without additional radiation exposure in an interventional procedure. Further, electronic collimation, if available, which overlays a collimator blade on the last image hold so that one can adjust field dimension without exposing the patient, also reduces radiation exposure (26). Image magnification either by means of geometric or electronic means, is considered useful. However, the ability to create magnified images in almost all cases results in a higher radiation exposure dose. Moving the source closer to the patient or the receptor further away can magnify the image. Thus, it is best to minimize the geometric magnification in prolonged procedures by keeping the image receptor close to the patient and the source away. Most modern fluoroscopes can also magnify the image electronically within the image intensifier. Some modern systems typically have at least three and sometimes as many as five electronic magnification modes, each with a unique dose level. However, again, dose increases with greater electronic magnification.

Multiple dose level settings provided in various fluoroscopes also reduce the radiation exposure. A typical configuration is to provide three settings - low, medium and high- with the dose being half or twice the medium level at the low and high settings, respectively (26). Since the low dose setting tends to produce a very noisy image, whereas as a high dose setting produces low image noise, one should use medium dose setting for most procedures.

Pulsed fluoroscopy, available in most modern fluoroscopes, with the x-ray beam emitting as a series of short pulses rather than the continuous emission, could reduce radiation exposure significantly. With pulsed fluoroscopy, images may be acquired at 15 frames per second rather than the usual 30 frames per second. Each image is displayed multiple times in sequence to provide a 30 frames per second display. Aufrichtig et al (47) showed average dose savings of $22 \%$, $38 \%$, and $40 \%$ at 15,10 , and 7.5 frames per second, respectively. Pulsed fluoroscopy has a great advantage as long as the radiation exposure is lower at lower frame rates. However, if the tube current is set too high to achieve better quality images, the entire advantage of pulsed operation is defeated and there may be no actual dose savings (26). Others also have shown to reduce overall exposure by $20 \%$ to $75 \%$ during pulsed fluoroscopy $(48,49)$.

Decreasing radiation before it reaches the clinician's skin or body is a primary objective for safety for interventionalists. Since there is a significant amount of exposure to clinicians from scattered radiation coming from the patient, standing behind "lead barriers" whenever possible, using proper techniques and calibrated equipment, along with optimizing duration of exposure and distance from source are all "good practices." Proper shielding is a requirement in the fluoroscopy suit, even though no law mandates the use of all of the shielding devices available in practice today, including lead aprons, leaded gloves, thyroid shields, or protective eyewear. Lead aprons absorb between $90 \%$ and $95 \%$ of the scattered radiation that reaches them.

\section{CONCLUSION}

Fluoroscopy offers many advantages for performing precision diagnostic and therapeutic interventional procedures. The average exposure per procedure in this study shown is $7.7 \pm 0.21$ seconds with average exposure 
per patient of $13.2 \pm 0.33$ seconds. Extrapolated to 2,000 or 3,000 procedures a year, total exposure would be 15,400 seconds or 23,100 seconds. Exposure in terms of mREM would be 2,690 mREM outside the apron for 2,000 procedures with $0 \mathrm{mREM}$ inside the apron, whereas it would be 4.03 mREM outside the apron for whole body (head and trunk) effective dose equivalent exposure with $0 \mathrm{mREM}$ per year inside the apron. Based on the outside apron doses, if one performs 4,000 procedures without protection they would cross the annual limit. However, this is much less than the annual limit exposure described for lens of the eye (15 mREM per year), extremities or skin (50 mREM per year). This study has shown that appropriate utilization of dose reduction techniques provide a safe environment. It is concluded that it is feasible to perform all procedures under fluoroscopy in the present setting described safely and effectively.

\section{ACKNOWLEDGMENTS}

The authors wish to thank Tonie Hatton, Marla Neihoff and Lorie Caldwell for their assistance in preparation of this manuscript.

\section{REFERENCES}

1. Rathmell JP. Imaging in regional anesthesia and pain medicine: We have much to learn. Reg Anesth Pain Med 2002; 27:240-241.

2. Slipman CW. Pain physician embraces the future. Pain Physician 2002; 5:119-120.

3. Fishman SM, Smith H, Meleger A et al. Radiation safety in pain medicine. Reg Anesth Pain Med 2002; 27:296-305.

4. Lenrow DA, Bhagia SM, Slipman CW. Radiation safety in interventional pain management. In Manchikanti L, Slipman CW, Fellows B (eds), Interventional Pain Management: Low Back Pain-Diagnosis and Treatment. ASIPP Publishing, Paducah, KY 2002; 541-546.

5. Manchikanti L, Bakhit CE, Pakanati RR, Fellows B. Fluoroscopy is medically necessary for the performance of epidural steroids. Anesthesia and Analgesia 1999; 89:1330-1331.

6. Manchikanti L, Singh V, Kloth D et al. Interventional techniques in the management of chronic pain: Part 2.0. Pain Physician 2001; 4:24-96.

7. Bogduk N. Epidural steroids for low back pain and sciatica. Pain Digest 1999; 9:226-227.

8. Manchikanti L. Transforaminal lumbar epidural steroid injections. Pain Physician 2000; 3:374-398.

9. Bogduk N. International Spinal Injection Society guidelines for the performance of spinal injection procedures. Part 1: Zygapophyseal joint blocks. Clin J Pain 1997; 13:285-302.
10. Manchikanti L, Singh V, Kloth D. Interventional pain management practice policies. In Manchikanti L (ed.), Interventional Pain Medicine: Documentation, Billing and Coding. ASIPP Publishing, Paducah, KY 2002; pp 169-222.

11. Manchikanti L. Definitions of interventional procedures. In Manchikanti L (ed.), Interventional Pain Medicine: Documentation, Billing and Coding. ASIPP Publishing, Paducah, KY 2002; pp 155-168.

12. Adminastar Federal Paravertebral Facet Joint Nerve Block (Diagnostic or Therapeutic) Policy. Policy Number AC-02-034.

13. Adminastar Federal Paravertebral Facet Nerve Denervation Policy. Policy Number AC-02-035.

14. Alabama Epidural Injection Policy. Policy Number AC-01-026.

15. Botwin KP, Thomas S, Gruber RD et al. Radiation exposure of the spinal interventionalist performing fluoroscopically guided lumbar transforaminal epidural steroid injections. Arch Phys Med Rehabil 2002; 83:697-701.

16. Botwin KP, Freeman ED, Gruber RD et al. Radiation exposure to the physician performing fluoroscopically guided caudal epidural steroid injections. Pain Physician 2001; 4:343-348.

17. Renfrew DL, Moore TE, Kathol MH, et al: Correct placement of epidural steroid injections: Fluoroscopic guidance and contrast administration. AJNR 1991; 12:1003-1007.

18. White AH, Derby R, Wynne G. Epidural injections for the diagnosis and treatment of low-back pain. Spine $1980 ; 5: 78-86$.

19. Manchikanti L, Bakhit CE, Pampati V. Role of epidurography in caudal neuroplasty. Pain Digest 1998; 8:277-281.

20. Stojanovic MP, Vu TN, Caneris $\mathrm{O}$ et al. The role of fluoroscopy in cervical epidural steroid injections: an analysis of contrast dispersal patterns. Spine 2002; 27:509-514

21. Price CM, Rogers PD, Prosser AS et al. Comparison of the caudal and lumbar approaches to the epidural space. Ann Rheum Dis 2000; 59:879-882.

22. Manchikanti L, Singh V. Caudal epidural use of steroids. In Manchikanti L, Slipman C, Fellows B (eds). Interventional Pain Management: Low Back Pain: Diagnosis and Treatment, ASIPP Publishing, Paducah, KY 2002 pp 277-312.

23. Hansen H. Fluoroscopy necessary for Sacral joint injection. Pain Physician 2002; 5: 4; 471.

24. Fredman B, Nun MB, Zohar E, et al. Epidural steroids for treating "failed back surgery syndrome": Is fluoroscopy really necessary? Anesth Analg 1999; 88:367-372.

25. Manchikanti L, Bakhit CE, Pakanati RR et al. Fluoroscopy is medically necessary for the performance of epidural steroids. Anesth Analg 1999; 89:13301331. 
26. Mahesh M. Fluoroscopy: Patient radiation exposure issues. Radiographics 2001; 21:1033-1045.

27. Shope TB. Radiation-induced skin injuries from fluoroscopy. Radiographics 1996; 16:1195-1199.

28. Wagner LK, Archer BR. Minimizing risks from fluoroscopic $\mathrm{x}$-rays: Bioeffects, instrumentation, and examination, partners in radiation management, Houston TX, 1998.

29. United States Food and Drug Administration. Public Health Advisory: Avoidance of Serious X-ray Induced Skin Injuries to Patients During FluoroscopicallyGuided Procedures. Rockville, MD, Center for Devices and Radiological Health, United States Food and Drug Administration, September, 1994.

30. Paulson EK, Sheafor DH, Enterline DS et al. CT fluoroscopy - guided interventional procedures: Techniques and radiation dose to radiologists. Radiology 2001; 220:161-167.

31. Archer BR, Wagner LK. Protecting patients by training physician in fluoroscopic radiation management. $J$ Appl Clin Med Phys 2000; 1:32-37.

32. Sovik E, Klow NE, Hellesnes J et al. Radiation-induced skin injury after percutaneous transluminal coronary angioplasty: Case report. Acta Radiol 1996; 37:305306.

33. Knautz MA, Abele DC, Reynolds T. Radiodermatitis after transjugular intrahepatic portosystemic shunt. South Med J 1997; 90:352-356.

34. Lichtenstein DA, Klapholtz L, Vardy DA et al. Chronic radiodermatitis following cardiac catheterization. Arch Dermatol 1996; 132:663-667.

35. Rosenthal LS, Beck TJ, Williams JR et al. Acute radiation dermatitis following radiofrequency catheter ablation of atrioventricular nodal reentant tachycardia. Pacing Clin Electrophysiol 1997; 20:1834-1839.

36. Vano E, Gonzalez L, Beneytez F et al. Lens injuries induced by occupational exposure in non-optimized interventional radiology laboratories. Br J Radiol 1998; 71:728-733.

37. Racz GB. Foreword (eds). Interventional Pain Management: Low Back Pain: Diagnosis and Treatment, ASIPP Publishing, Paducah, KY 2002, pp i-ii.
38. National Council on Radiation Protection and Measurements (NCRP). Report No. 116 Limitation of Exposure to Ionizing Radiation, Bethesda, MD: NCRP Publications, 1993.

39. International Commission on Radiological Protection Recommendations of the International Commission on Radiological Protection, ICRP Publication, No. 60, Ann ICRP 21 (1-3), 1991.

40. Manchikanti L, Pampati V. Research designs in interventional pain management: Is randomization superior, desirable or essential? Pain Physician 2002; 5:275-284.

41. Statkiewicz-Sherer MA, Viscanti PJ, Ritenour ER. Radiation Protection in Medical Radiography, Third edition, Mosby, St. Louis, 1998.

42. Bushong S. Radiologic Science for Technologists: Physics, Biology, and Protection, Sixth edition, Mosby, St. Louis, 1997.

43. Boone JM, Levin DC. Radiation exposure to angiographers under different fluoroscopic imaging procedures. Radiology 1991; 180:861-865.

44. Gray JE, Swee RG. The elimination of grids during intensified fluoroscopy and photofluoro spot imaging. Radiology 1982; 144:426-429.

45. Drury P, Robinson A. Fluoroscopy without the grid: A method of reducing the radiation dose. Br J Radiol 1980; 53:93-99.

46. Wilson DL, Xue P, Aufrichtig R. Perception of fluoroscopy last-image hold. Med Phys 1994; 21:1875-1883

47. Aufrichtig R, Xue P, Thomas CW et al. Perceptual comparison of pulsed and continuous fluoroscopy. Med Phys 1994; 21:245-256.

48. Adelstein SJ. Uncertainty and relative risks of radiation exposure. JAMA 1987; 258:655-657.

49. Hernandez RJ, Goodsitt MM. Reduction of radiation dose in pediatric patients using pulsed fluoroscopy. Am J Roentgerol 1996; 167:1247-1253. 